\title{
Nanoone Woman Underwear for Dysmenorrhea
}

\section{Management}

\author{
Choo-Aun $\mathrm{Neoh}^{1}$, Lan-Shiang $\mathrm{Wu}^{2}$ and Karen $\mathrm{Neoh}^{3}$ \\ 1. Department of Pain Clinic, Pingtung Christian Hospital, Pingtung 900, Taiwan \\ 2. Department of Obstetrics \& Gynecology, Pingtung Christian Hospital, Pingtung 900, Taiwan \\ 3. University of Traditional Chinese Medicine, Jinan, Shandong 250000, China
}

\begin{abstract}
Objective: The objective of this study is to evaluate the effectiveness of Nanoone Woman Underwear used in the management of dysmenorrhea. Methods: The purpose is to evaluate the mean change in menstrual pain intensity during menstrual cycles from baseline to after wearing Nanoone Woman Underwear in women with primary dysmenorrhea. The severity of dysmenorrhea pain experienced by subjects will be evaluated on a VAS (visual analogue scale), ranging from zero (no pain) to ten (very severe pain). Effectiveness will be evaluated at baseline and subsequently on study weeks 1-2, 5-6 and 9-10 using a VAS. The primary objective is to evaluate the mean change in menstrual pain intensity at the endpoint visit from baseline. The difference of the mean change in menstrual pain intensity during each menstrual cycle from baseline will also be investigated. Subjects will be asked to use the VAS scoring system to record, on a provided sheet, their experienced menstrual pain level daily during menstrual bleeding for a total of three consecutive menstrual cycles in house. Results: The mean maximum pain level at poster menstrual cycle was more significant lower than at prior menstrual cycle when respective compared with experienced menstrual pain intensity based on the result of statistical analysis. Conclusion: The mean change in maximum pain level at menstrual cycle was significant lower after long wearing Nanoone Woman Underwear in women with primary dysmenorrheal. Therefore, the improvement extent of menstrual pain intensity was more significant after long wearing Nanoone Woman Underwear.
\end{abstract}

Key words: Dysmenorrhea, nanoone technology, pain, underwear.

\section{Introduction}

Dysmenorrhoea, or painful menses, has been reported to be highly prevalent among young women around the world. Primary dysmenorrhea refers to painful menstrual periods in the absence of any underlying pathology. Dysmenorrhea is a very common problem among young women, and can occur in up to $50 \%$ of menstruating women. Dysmenorrhea can be classified as either primary or secondary based on the absence or presence of an underlying cause. Secondary dysmenorrhea is dysmenorrhea which is associated with an existing condition. The most common cause of secondary dysmenorrhea is endometriosis. Other causes include leiomyoma,

Corresponding author: Choo-Aun Neoh, Ph.D., research fields: anesthesia, pain management, acupuncture, traditional Chinese medicine, hospice care, health promotion and community health. E-mail: neohca@gmail.com. adenomyosis, ovarian cysts, and pelvic congestions. The major cause of primary dysmenorrhea could be classified as unknown cause, which leads to uterine spasm and strong secretion of prostaglandins. There are three approaches to the management of primary dysmenorrhea: pharmacological, non-pharmacological and surgical [1, 2].

According to some experimental research in medical journals, the effectiveness of "negative ions" in the air generally have been identified as follows: blood purification, activate cells, adjustment of autonomic nervous system, increment resistibility, clean air and antibacterial, control inflammation, pain and anticancer. Chen Yi Enterprise, Co., Ltd. designed a negative ion material that can supply delocalized electron. It is then added fiber inside, make clothes to wear over the body. No external power or battery needed, only depend on 
infrared body photon hit of thermoelectric effect or heart compression pulse, blood flow of piezoelectric, therefore can sustained sufficient amount of effective negative ion, to eliminate positive ions in vivo, or make oxygen free radical to obtain the lack electrons. It can decrease active free radical and get non toxic effect in short time $[3,4]$.

"Nanoone" negative ion of textiles, which is a healthy material specifically designed for human body in short distance and can continue to produce negative ion for long duration. Our human bodies need the "negative ions" to neutralize free radical in the human body. This product's raw materials have been certified in Europe and America rigorous testing and certification unit, fully compliant with EU standards of the latest textile import controls and CPSIA (Consumer Product Safety Improvement Act). Long-term wear was with no negative adverse effects and toxicity, and there was no any toxic detected from babies born within 36 months after wearing it [3].

The objective of this study is to evaluate the effectiveness of Nanoone Woman Underwear used in the management of dysmenorrhea. The changes in menstrual pain intensity during menstrual cycles after wearing Nanoone Woman Underwear in women with primary dysmenorrhea were observed. The severity of dysmenorrhea pain experienced by subjects will be evaluated on a VAS (visual analogue scale), ranging from zero (no pain) to ten (very severe pain). The VAS has been previously demonstrated to be sensitive measure relative to pharmacological and non-pharmacological procedures that may alter the experience of pain and VAS scores have been demonstrated to correlate highly with pain measured on a verbal numeric rating scale $[1,3,5]$.

\section{Methods}

The purpose is to evaluate the mean change in menstrual pain intensity during menstrual cycles from baseline to after wearing Nanoone Woman Underwear in women with primary dysmenorrhea. The
Institutional Review Board of Pingtung Christian Hospital, Ping Tung County, Taiwan, approved the research, and all subjects were included only after they signed agreement on inform consent form given to them. The research is registered in ClinicalTrials.gov. The study is expected to be completed when a total number of valuable subjects reached at least 40 .

Subjects must fulfill all of the following criteria to be eligible to enter the study:

(1) Females aged $\leq 40$ years old.

(2) Screened by inquiry and diagnosed as primary dysmenorrhea by gynaecologist with pelvic ultrasonography.

(3) Female subject who:

- is using adequate contraception since last menstruation and no plan for conception during the study;

- is non-lactating;

- has negative pregnancy test (urine) within 14 days prior to the study.

(4) Informed consent form signed.

Subjects with any of the following criteria are excluded from the study:

(1) Sensitivity to study product.

(2) Patient has been diagnosed with secondary dysmenorrhea (defined as identifiable pelvic pathology).

(3) Patient has clinically significant physical disability or abnormal findings on physical examination or laboratory testing judged by the investigator or co-investigator.

(4) Participation of any clinical investigation during the last 30 days.

(5) Individuals are judged by the investigators or co-investigator to be undesirable as subjects.

Subjects were selected among Taiwanese residents, and were screened for adequacy to enroll the study. All subjects signed an ICF (inform consent form). A copy of the signed ICF was handed to the subjects upon enrollment. The subject must be qualified for the study by fulfilling all of the inclusion and none of the 
exclusion criteria. Laboratory values which are out of reference range will not qualify unless the physician deems the deviation NCS (not clinically significant). In case of serious adverse events or other unforeseen circumstances, the complete study may, after consultation with the sponsor, be discontinued by the investigator, if this appears necessary for medical or ethical reasons. The following circumstances may lead to discontinuation of the study by an individual subject who will then be recorded as a drop-out:

(1) Withdrawal for personal reasons;

(2) Adverse events necessitating withdrawal from the study;

(3) Sudden incidence of diseases;

(4) Circumstances in which the health of the subject would be endangered upon continued participation in the study;

(5) Protocol violation.

Drop-outs will be replaced respectively to achieve at least 40 subjects completed the whole study. In case of drop out after wearing of study product a final examination should be performed whenever possible. This is an open-label study design. Subjects were required to wear the Nanoone Woman Underwear daily during menstrual bleeding for a total of three consecutive menstrual cycles. Table 1 is the events and time schedule.

The severity of dysmenorrhea pain experienced by subjects was evaluated on a VAS (visual analogue scale), ranging from zero (no pain) to ten (very severe pain). History of dysmenorrhea was also self-reported the intensity of experienced menstrual pain. Subjects were asked to use the VAS scoring system to record, on a provided sheet, their experienced menstrual pain level daily during menstrual bleeding for a total of three consecutive menstrual cycles in house. Subjects were allowed to continue taking pharmacological pain relief if required and it should be recorded. The study period for each subject will at least for 70 days. After enrolled into the study, the study day 1 is the first menstrual cramps of each subject. And if the next menstrual cycle has delayed for more than 10 days, the pregnancy test must be performed to ensure the physical condition of subject, and the visit date (day and week) will be corrected with the following menstrual cramps. A subject was required to make a total of 7 visits in the study period. Patients' routinely used medications or treatments for other indications which are judged by the investigator or co-investigator as not to affect the effectiveness and safety assessments in this study are permitted. Any use of drugs will be documented in the CRF (case report forms), specifying the substance, dose, time and reason for use. Demographic data (e.g., subject's initials, sex, date of birth, height and weight), medical history, and the related treatment will be recorded. Subjects give the signed informed consent form will be screened.

Table 1 Events and time schedule.

\begin{tabular}{|c|c|c|c|c|c|c|c|}
\hline \multirow{2}{*}{$\begin{array}{l}\text { Period } \\
\text { Visit }\end{array}$} & \multirow{2}{*}{$\begin{array}{l}\text { Screening/Baseline } \\
\text { V } 1\end{array}$} & \multicolumn{6}{|c|}{ Treatment } \\
\hline & & V 2 & V 3 & V 4 & V 5 & V 6 & V 7 \\
\hline Day (D) & $\mathrm{D}-28 \sim 0$ & D $1 \sim 7$ & D $8 \sim 14$ & D 29 35 & D 36 42 & D 57 63 & D $64 \sim 70$ \\
\hline Week (W) & $\mathrm{W}-4 \sim 0$ & W 1 & W 2 & W 5 & W6 & W 9 & W 10 \\
\hline Medical history & $\bullet$ & & & & & & \\
\hline Clinical examination & 0 & & & & & & - \\
\hline Laboratory measurement & ○ & & & & & & ○ \\
\hline Study product & & ○ & & ○ & & ○ & \\
\hline VAS evaluation & ○ & 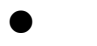 & & 0 & & 0 & \\
\hline Inquiry* & ○ & & ○ & & 0 & & ○ \\
\hline Vital sign measurement & ○ & & ○ & & ○ & & ○ \\
\hline Safety measurement & & & ○ & & ○ & & ○ \\
\hline
\end{tabular}

*: Study product will be provided after each inquiry at visit 1, visit 3 and visit 5 . 
The clinical examination includes vital signs and physical examination. Blood pressure and heart rate in the sitting position, body weight and height were measured. A blood sample was taken at prestudy screening and visit 7 in order to perform the laboratory measurements. If subjects have performed any item of the laboratory tests within two month before entering the study, the item was not performed at prestudy screening. The abnormal observation was recorded on the CRFs and the evaluation of clinical significance was judged by the investigators. For early termination, the most possible efforts were made to encourage the subject to complete the final laboratory test. Table 2 is the laboratory measurements.

The severity of dysmenorrhea pain experienced by subjects will be evaluated on a VAS, ranging from zero (no pain) to ten (very severe pain). The VAS has been previously demonstrated to be sensitive measure relative to pharmacological and non-pharmacological procedures that may alter the experience of pain and VAS scores have been demonstrated to correlate highly with pain measured on a verbal numeric rating scale. Effectiveness will be evaluated at baseline and subsequently on study weeks 1-2, 5-6 and 9-10 using a VAS. The primary objective is to evaluate the mean change in menstrual pain intensity at the endpoint visit from baseline. The difference of the mean change in menstrual pain intensity during each menstrual cycle from baseline will also be investigated. Subjects will be asked to use the VAS scoring system to record, on a provided sheet, their experienced menstrual pain level daily during menstrual bleeding for a total of three consecutive menstrual cycles in house. Safety will be evaluated by the clinically significant changes in terms of:

(1) physical examination;
(2) vital signs;

(3) laboratory examination parameters;

(4) incidence of adverse events.

Heart rate, systolic and diastolic blood pressure and body temperature were measured and recorded in sitting position at the following times: Study Day 28-0, 8-14, 36-42 and 64-70. Within 7 days after completion of the clinical part, a final examination including hematology and biochemistry tests as carried out for subject inclusion were performed in order to determine possible changes in the subject's state of health. Safety evaluation included changes in laboratory data, vital signs, physical examination parameters and all adverse events that may have happened during the study.

Data analyses and summaries of the safety endpoints will be performed for the following populations:

(1) ITT (intention-to-treat) population - All subjects who receive at least once of study product and have at least one post-baseline assessment for the objective variable regardless of their compliance with the protocol.

(2) PP (per-protocol) population-All subjects who using the study product and who complete the study without any major protocol deviation.

(3) Safety population-All subjects who receive at least once of the study product. Safety endpoints will be analyzed on the safety population.

\section{Results}

Total numbers of 44 subjects enrolled into the study. During the study period, two subjects withdrew from the study due to personal reasons, and they were not replaced because the expected subject numbers to be completed the whole study (at least 40) should be

Table 2 Laboratory measurements.

\begin{tabular}{ll}
\hline Item & Statement \\
\hline Hematology & Hemoglobin, hematocrit, WBC count, RBC count and platelet count. \\
Biochemistry & SGOT (AST), SGPT (ALT), creatinine, uric acid and BUN. \\
Pregnancy test & Urinary pregnancy test will be carried out only for female subject. \\
\hline
\end{tabular}


enough. Therefore, the total numbers of subjects completed the entire study were 42 . Only the data obtained from the subjects who complete the whole study will be reported. Table 3 is demographic characteristics.

The mean intensity of experienced menstrual pain at baseline was $6.76 \pm 1.51$. The mean maximum pain levels at first menstrual cycles, second menstrual cycles and third menstrual cycles were $5.95 \pm 2.48$, $5.48 \pm 2.35$ and $5.02 \pm 2.19$, respectively.

According to the result of statistical analysis, the mean maximum pain level at first menstrual cycle were already significant lower after wearing Nanoone Woman Underwear in women with primary dysmenorrheal compared with experienced menstrual pain intensity. The mean maximum pain level at second menstrual cycle and third menstrual cycle were consistent significant lower after wearing Nanoone Woman Underwear compared with experienced menstrual pain intensity. The mean maximum pain level at third menstrual cycle was more significant lower than at second menstrual cycle when respective compared with experienced menstrual pain intensity based on the result of statistical analysis in Table 4 .

On the primary objective of this study, result of the mean change in menstrual pain intensity at the endpoint visit from baseline, was described as follows. The difference of mean change in maximum pain level at each menstrual cycle from baseline experienced menstrual pain intensity was analyzed and listed in Table 3. The mean change in maximum pain level at first menstrual cycle, second menstrual cycle and third menstrual cycle from baseline experienced menstrual pain intensity were $-0.810 \pm 2.04,-1.29 \pm 1.81$ and $-1.74 \pm 2.24$, respectively. In accordance with the result of statistical analysis (show in Table 5), the mean change in maximum pain level at third menstrual cycle (the endpoint visit) from baseline experienced menstrual pain intensity was significant lower than at first or second menstrual cycle. Therefore, the improvement extent of menstrual pain intensity is more significant after long wearing Nanoone Woman Underwear compared with short wearing Nanoone Woman Underwear in women with primary dysmenorrheal.

Table 3 Demographic characteristics.

\begin{tabular}{lll}
\hline \multicolumn{3}{c}{ Experimental group $(n=42)$} \\
\hline Characteristics & Mean & SD \\
\hline Age (years) & 22.5 & 4.31 \\
Height (cm) & 160.6 & 5.92 \\
Weight (kg) & 52.4 & 9.40 \\
Body mass index & 20.3 & 3.20 \\
History of dysmenorrhoea & 6.76 & 1.51 \\
\hline
\end{tabular}

Table 4 Difference of mean maximum pain level at each menstrual cycle from baseline.

\begin{tabular}{lllll}
\hline \multicolumn{5}{c}{ Pain level on VAS evaluation $(n=42)$} \\
\hline Baseline $($ mean \pm SD) & & Menstrual cycle $($ mean \pm SD) & $P$-value & \\
\hline \multirow{2}{*}{$6.76 \pm 1.51$} & First & $5.95 \pm 2.48$ & 0.01842 & $*$ \\
& Second & $5.48 \pm 2.35$ & 0.000017 & $*$ \\
& Third & $5.02 \pm 2.19$ & 0.000007 & $*$ \\
\hline
\end{tabular}

*: Significant difference compared each menstrual cycle with baseline.

By paired-sample $t$-test

Table 5 The mean change in maximum pain level at each menstrual cycle from baseline.

\begin{tabular}{lcc}
\hline \multicolumn{2}{c}{ Mean change in pain level on VAS evaluation $(n=42)$} \\
\hline Each menstrual cycle compare with baseline (mean \pm SD) & $P$-value \\
\hline First & $-0.810 \pm 2.04$ & - \\
Second & $-1.29 \pm 1.81$ & 0.098237 \\
Third & $-1.74 \pm 2.24$ & 0.018555 \\
\hline
\end{tabular}

*: Significant difference compared third cycle with baseline and first cycle with baseline.

By paired-sample $t$-test 


\section{Conclusions}

To sum up these results, the effectiveness of Nanoone Woman Underwear using in the management of dysmenorrhea has been observed. The mean change in maximum pain level at menstrual cycle was significant lower after long wearing Nanoone Woman Underwear in women with primary dysmenorrheal. Therefore, the improvement extent of menstrual pain intensity was more significant after long wearing Nanoone Woman Underwear. The longer wearing time of Nanoone Woman Underwear, the better improvement proportion of menstrual pain had been observed. The mean degree of menstrual pain during first menstrual cycle, second menstrual cycle and third menstrual cycle had a decrease tendency. Furthermore, demand for pharmacological pain relief treatment was decreased when Nanoone Woman Underwear using in the management of dysmenorrheal.

The safety of Nanoone Woman Underwear using during each menstrual cycle was monitored throughout the study. There was no any adverse event during the study. It indicates that, Nanoone Woman Underwear using in the management of dysmenorrheal is helpful and safe for dysmenorrheal women. Long wearing of Nanoone Woman Underwear for dysmenorrheal women may offer benefits in the management of dysmenorrheal.
In addition, there are various opinions about improving dysmenorrhoea. Hence, we also propose our opinions, and hope to provide more studies to support our argument.

\section{Acknowledgments}

The authors wish to thank the women participating in the study and Pingtung Christian Hospital, Taiwan and support source is Red Pharma \& Biotech Research Corporation and First Biotech Textile International Corp..

\section{References}

[1] J.F. Cheng, Z.J. Lu, Y.C. Su, L.C. Chiang, R.Y. Wang, A traditional Chinese herbal medicine used to treat dysmenorrhoea among Taiwanese women, Journal of Clinical Nursing 17 (19) (2008) 2588-2595.

[2] C.A. Smith, C.A. Crowther, O. Petrucco, J. Beilby, H. Dent, Acupuncture to treat primary dysmenorrhea in women: A randomized controlled trial, Evidence-Based Complementary and Alternative Medicine 2011 (2011) $1-11$.

[3] Product information of NANOONE, Chen Yi Enterprise, Co., Ltd., Taiwan, 2011, pp. 45. (in Chinese)

[4] M. Terman, J.S. Terman, Controlled trial of naturalistic dawn simulation and negative air ionization for seasonal affective disorder, The American Journal of Psychiatry 163 (12) (2006) 2126-2133.

[5] L.L. Yeh, J.Y. Liu, K.S. Lin, Y.S. Liu, J.M. Chious, K.Y. Liang, et al., A randomised placebo-controlled trial of a traditional Chinese herbal formula in the treatm ent of primary dysmenorrhoea, PLoS ONE 2 (8) (2007) 1-11. 